\title{
Narcolepsy associated with the 2009 H1N1 pandemic in China
}

Research conducted in a Chinese patient population has provided evidence for a link between peaks of onset of the chronic sleep disorder narcolepsy and seasonal patterns of upper airway infections; in particular, the $2009 \mathrm{H} 1 \mathrm{~N} 1$ influenza pandemic.

The study was prompted by two observations. "First, we noticed that many patients who had recently developed narcolepsy had had streptococcus throat infections," say senior investigator Emmanuel Mignot, Stanford University Medical Center, California, USA. "Second, we started to see more and more children, and we noticed at Stanford and in China that most of the cases that came to see us had disease onset during the spring."

Mignot's group teamed up with Fang Han and colleagues at Beijing University People's Hospital, China to study a cohort of 629 patients - predominantly children-all of whom had been diagnosed with narcolepsy between 1998 and 2010 in Beijing. Over this time period, the annual peak of narcolepsy onset was found to occur 5-7 months after the seasonal peak of upper airway infections. Most strikingly, the $2009 \mathrm{H} 1 \mathrm{~N} 1$ pandemic was followed by a threefold rise in the incidence of narcolepsy compared with previous years.

In northern Europe, cases of narcolepsy have recently been linked to the $\mathrm{H} 1 \mathrm{~N} 1$ vaccine, raising concerns over its safety. However, only $5.6 \%$ of the patients in the Chinese study recalled receiving the vaccine, so narcolepsy was unlikely to be attributable to vaccination in this population.

Narcolepsy is caused by the loss of hypocretin neurons from the hypothalamus. Mignot and colleagues propose that upper airway infections could trigger an immune response that ultimately leads to destruction of these cells, although the precise mechanisms remain to be elucidated. Mignot points

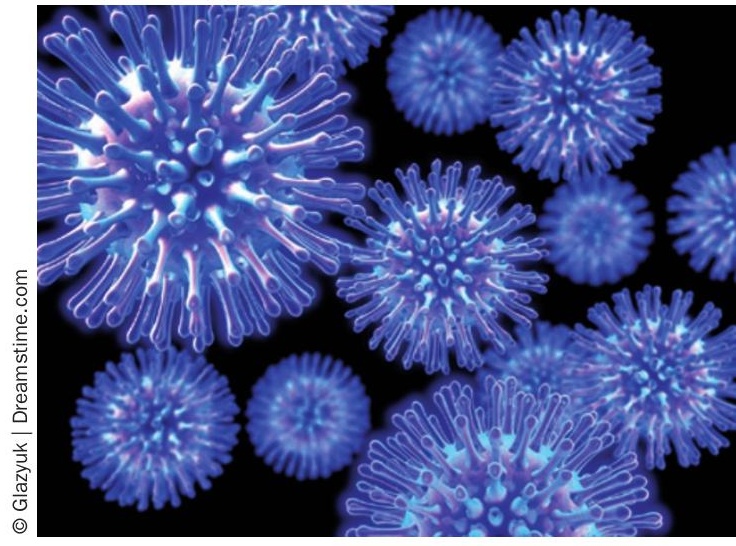

out that an increased frequency of neurological disorders was observed after the 1918 Spanish influenza pandemic, and he suggests that narcolepsy could share common pathological mechanisms with other conditions such as Parkinson disease and Sydenham chorea.

Heather Wood

Original article Han, F. et al. Narcolepsy onset is seasonal and increased following the $2009 \mathrm{H} 1 \mathrm{~N} 1$ pandemic in China. Ann. Neurol. 70, 410-417 (2011) 